countries which previously had reported very high rates. These declines are probably due to changes in healthcare systems, diagnostic capacity and reporting rather than true changes in the incidence. However, dramatic increases were noted in other countries and-based on the information from the male-to-female ratio-this is most likely due to recent increases of syphilis among MSM. The overall trend in chlamydia showed a continuously increasing trend, reflecting an increase in testing and screening practices across countries. These trends must be interpreted with caution due to the heterogeneity in reporting and healthcare systems. A further limitation to the interpretation is that many diagnoses are either not made or under-reported. Diagnoses from certain countries cannot be included in trend analyses as they do not have comprehensive surveillance for STI.

Conclusion Enhanced surveillance of STI in Europe is essential to provide the information that is necessary to monitor the distribution of disease and to evaluate the public health response to control the transmission of infections. Collaborating within the European STI expert networks provides the platform for sharing best practices and expert knowledge across Europe.

\section{S15.2 EUROPEAN GONOCOCCAL ANTIMICROBIAL SURVEILLANCE PROGRAMME (EURO-GASP): TOWARDS TIMELIER MONITORING}

doi:10.1136/sextrans-2011-050102.63

${ }^{1} \mathrm{M} J$ Cole, ${ }^{1} \mathrm{~S}$ A Chisholm, ${ }^{2} \mathrm{M}$ Unemo, ${ }^{3} \mathrm{~S}$ Hoffmann, ${ }^{4} \mathrm{M} \mathrm{J}$ W van de Laar, ${ }^{1} \mathrm{C}$ A Ison. ${ }^{1}$ Health Protection Agency, London, UK; ${ }^{2}$ Örebro University Hospital, Örebro, Sweden; ${ }^{3}$ Statens Serum Institut, Copenhagen, Denmark; ${ }^{4}$ European Centre for Disease Prevention and Control, Stockholm, Sweden

Background It is imperative that antimicrobial resistance (AMR) surveillance systems work in a timely manner and outcomes are translated into treatment guidelines without delay. For the first time in 2010, the European gonococcal antimicrobial surveillance programme (Euro-GASP) introduced biannual testing to allow more frequent reporting of trends and developments in Neisseria gonorrhoeae AMR across Europe.

Methods In the first half of 2010, 18 out of the 30 EU/EEA Member States contributed up to 55 consecutive gonococcal isolates to EuroGASP. Susceptibility testing was performed by Etest or agar dilution for therapeutically relevant antimicrobials: azithromycin, cefixime, ceftriaxone, ciprofloxacin, gentamicin and spectinomycin. Biannual testing continued into the second half of 2010. Additionally 10 laboratories performed decentralised testing with the submission of AMR data into Euro-GASP instead of gonococcal isolates.

Results In the first round of biannual testing $900 \mathrm{~N}$ gonorrhoeae isolates were tested. Eight per cent of the isolates displayed decreased susceptibility $(>0.125 \mathrm{mg} / \mathrm{l})$ to cefixime, which was an increase from $5 \%$ in 2009 . No decreased susceptibility to ceftriaxone was detected. For ciprofloxacin, the overall incidence of resistant isolates decreased from $63 \%$ in 2009 to $52 \%$ in 2010 and azithromycin resistance decreased from $13 \%$ in 2009 to $5 \%$ in 2010 . No resistance to spectinomycin was demonstrated. The MIC50 and MIC90 of gentamicin in the isolates remained at $8 \mathrm{mg} / \mathrm{l}$ in 2010 .

Conclusions The results from the first round of biannual testing have shown that isolates displaying decreased susceptibility to cefixime are rapidly increasing. This is a major concern as cefixime is a widely used, recommended therapy for gonorrhoea across Europe. Healthcare professions should be aware of cefixime treatment failures, particularly in light of the recent identification of treatment failures in Norway and the UK. The MICs of ceftriaxone need to be monitored continually to preserve this precious treatment option. Euro-GASP is currently being adjusted to allow for timely reporting and decentralised testing including representativeness and links to epidemiological data. All of which contribute to monitoring the ever changing gonococcus in timelier and more efficient ways, with the ultimate aim of treating infected individuals successfully.

\section{S15.3 EUROPEAN MSM INTERNET SURVEY (EMIS): DIFFERENCES IN SEXUALLY TRANSMISSIBLE INFECTION TESTING IN EUROPEAN COUNTRIES}

doi:10.1136/sextrans-2011-050102.64

${ }^{1} \mathrm{U}$ Marcus, ${ }^{1} \mathrm{~A} J$ Schmidt, ${ }^{2} \mathrm{M}$ Breveglieri, ${ }^{3} \mathrm{P}$ F Davila, ${ }^{3} \mathrm{~L}$ Ferrer, ${ }^{3} \mathrm{C}$ Folch, ${ }^{4} \mathrm{~F}$ Hickson, ${ }^{5} \mathrm{H} J$ Hospers, ${ }^{2} \mathrm{M}$ Mirandola, ${ }^{4} \mathrm{D}$ Reid, ${ }^{4} \mathrm{P}$ Weatherburn, for the EMIS network. ${ }^{1}$ Department for Infectious Disease Epidemiology, Robert Koch Institute, Berlin, Germany; ${ }^{2}$ Regional Centre for Health Promotion, ULSS 20-Veneto Region, Verona, Italy; ${ }^{3}$ Centre for Epidemiological Studies on HIVIAIDS in Catalonia (CEEISCAT), Barcelona, Spain; ${ }^{4}$ Sigma Research, London School of Hygiene and Tropical Medicine, UK; ${ }^{5}$ Maastricht University, University College Maastricht, The Netherlands

Background Comparing rates of sexually transmissible infections (STIs) among men who have sex with men (MSM) in different European countries is challenging due to national differences in reporting systems, healthcare systems, infectious disease surveillance methods, quality of data, and/or levels of social acceptance of homosexual behaviours and openness about homosexuality.

Methods From June through August 2010, the European MSM Internet Survey (EMIS) mobilised more than 180000 respondents from 38 European countries to complete an online questionnaire in one of 25 languages. The questionnaire covered sexual happiness, HIV and STI-testing and diagnoses, unmet prevention needs, intervention performance, HIV-related stigma and gay-related discrimination. Recruitment was organised predominantly online, through gay social media, and links and banners on more than 100 websites for MSM all over Europe.

Results Perceptions on access to free/affordable STI-testing differed across Europe (median: 80\%; range: $40-95 \%$ ); and was substantially correlated with reported recent STI-testing $\left(R^{2}=0.27\right)$. Quality of STI-testing was highly diverse: While blood-testing was common in all participating countries, only Ireland, Malta, and the UK seem to offer penile or particularly anal examinations as standard of care. In all participating countries HIV-positive respondents reported higher rates of both STI-testing and diagnosis. Self-reported STI-screening among men without HIV diagnosis ranged from 10\% (Turkey) to $37 \%$ (Netherlands). Substantial correlations between rates of testing procedures appropriate for MSM (such as anal or genital swabs) and diagnosed gonorrhoea $\left(R^{2} H I V-p o s=0.24\right)$ or Chlamydia infections were observed $\left(R^{2} H I V\right.$-pos $=0.50 ; R^{2}$ others $\left.=0.29\right)$.

Conclusion Self-reported testing and diagnosis rates for bacterial STIs suggest high levels of under-diagnosis and unmet sexual healthcare needs in most European countries. In Europe, there is an urgent need to implement or improve sexual healthcare tailored to MSM-specific needs.

\section{S15.4 RE-EMERGENCE OF LYMPHOGRANULOMA VENEREUM IN EUROPE AND THE PUBLIC HEALTH RESPONSE}

doi:10.1136/sextrans-2011-050102.65

${ }^{1} \mathrm{H}$ Ward, ${ }^{2} \mathrm{H} \mathrm{J} \mathrm{C}$ de Vries, ${ }^{3} \mathrm{M}$ van de Laar. ${ }^{1}$ Imperial College, London, UK; ${ }^{2} \mathrm{GGD}$ Amsterdam, The Netherlands; ${ }^{3}$ European Centre for Disease Prevention and Control, Stockholm, Sweden

Background In January 2004, public health officials in the Netherlands noted an outbreak of cases of lymphogranuloma venereum (LGV) among men who have sex with men (MSM). Since then a number of outbreaks and cases have been reported from European countries, North America and Australia. The re-emergence of LGV in 